\title{
Résumés de thèses
}

\section{Amélioration génétique des Eucalyptus tropicaux. Contribution majeure à la foresterie clonale}

\author{
B. MARTIN ${ }^{(*)}$
}

La thèse présente une succession de dix publications de 1971 à 1987, groupées autour du thème central de l'amélioration génétique des Eucalyptus tropicaux. La plupart des travaux ont été réalisés à Pointe-Noire (Congo) dans le cadre du Centre Technique Forestier Tropical (C.T.F.T.) et de l'Unité l'Afforestation Industrielle du Congo (U.A.I.C.). Beaucoup de références viennent également des plantations de la Société Aracruz Florestal S.A. au Brésil (Espirito Santo).

La présentation de l'ensemble des documents est réalisée sous forme d'une étude de synthèse utilisant également toute la bibliographie. Cette étude s'articule en sept chapitres.

\section{Intérêt du genre Eucalyptus}

Le genre Eucalyptus, très diversifié (600 espèces), est limité à l'Australie et la Tasmanie, mais quelques espèces se retrouvent également dans les îles indo-malaises, au nord, où on trouve deux espèces qui n'existent pas sur le continent australien : ce sont l'Eucalyptus urophylla des îles de la Sonde et l'Eucalyptus deglupta qui s'étend de la Papouasie-Nouvelle Guinée aux Philippines.

Après un aperçu de la paléogéographie et de la répartition actuelle, on peut y trouver les principaux traits morphologiques et les grandes lignes de la classification. La végétation australienne est décrite et en particulier les types forestiers, ainsi que les limites écologiques du genre Eucalyptus.

Une revue des diverses utilisations du bois et des produits secondaires du genre est présentée.

\section{Etude de la variatiblité du genre Eucalyptus}

La notion d'espèce est assez floue chez ce genre diversifié. Les espèces tropicales sont moins nombreuses que les espèces tempérées ou subtropicales.

$\left(^{*}\right)$ Thèse soutenuc le 26 mai 1987 à l'Université de Paris-Sud (Centre dOrsay) par Bernard Martin, professeur de Génétique et Reboisement au centre de Nancy de l'Ecole Nationalc du G.R.E.F., pour le titre de Docteur-en-sciences. 
Vingt trois espèces pour les climats tropicaux humides et 33 espèces pour les climats tropicaux secs sont mentionnés. Une présentation plus précise est faite pour les dix principales espèces tropicales (E. deglupta, E. urophylla, E. alba, E. cloeziana, E. tereticornis, E. torelliana, E. grandis, E. camaldulensis, E. microtheca et E. citriodora).

L'exploration des aires naturelles et la constitution des collections de semences ont été bien étudiées grầce aux importantes récoltes organisées par le C.T.F.T. en 1972 en Australie et en Indonésie, ce qui a permis une étude complète des Eucalyptus des îles de la Sonde.

Les problèmes liés aux essais d'espèces et les principaux résultats sont énoncés.

\section{Etude de la variabilité intraspécifique}

Les essais de provenances des principales espèces sont décrits non seulement au Congo, mais aussi dans les principaux pays utilisateurs (Afrique du Sud, NouvelleZélande, Brésil, Argentine, Burkina Faso, etc.). Les principales lois de variation sont énoncées en fonction de la latitude, de l'altitude et de la continentalité. Pour chacune des espèces principales, les provenances les plus vigoureuses et les plus stables sont citées.

Une interprétation est donnée pour la vigueur exceptionnelle et fréquente des provenances artificielles et les mesures conservatoires entreprises sont présentées.

\section{Amélioration par voie récurrente}

Le schéma classique a fait l'objet de plusieurs études, en particulier au Congo, en Afrique du Sud, au Brésil et en Floride. Les résultats sont souvent décevants du fait du régime de reproduction de l'Eucalyptus qui présente toujours environ 25 p. 100 d'autofécondation, ce qui altère la descendance, les plants consanguins étant moins vigoureux, anormaux ou même létaux. D'autre part, les fécondations libres sont très variables selon les années et, de ce fait, les descendances sont irrégulières.

Les meilleurs résultats viennent d'hybridations intraspécifiques et les solutions restituant le plus vite possible un fort taux d'hétérozygotie, semblent les plus judicieuses.

Le cas de l'E. tereticornis et de l'E. urophylla au Congo et de l'E. grandis en Afrique du Sud et en Floride sont traités en détail.

\section{Les hydrides interspécifiques}

Alors qu'ils sont souvent contre-sélectionnés dans les aires naturelles et de ce fait assez rares, les hybrides interspécifiques sont par contre très fréquents dans l'aire artificielle. En tant qu'exotique, le genre Eucalyptus fabrique des hybrides interspécifiques d'autant plus fréquents et vigoureux que les milieux utilisés s'écartent davantage de celui des aires naturelles des espèces pures.

D'autre part, ils présentent souvent une meilleure adaptation. Au Congo en particulier, l'hybride E. alba $\times$ urophylla, appelé localement "PF1 " et l'hybride $E$. tereticornis $\times$ grandis présentent de forts avantages par rapport aux espèces pures. 
L'hybridation artificielle est traitée. Elle permet d'éviter les barrières phénologiques et élargit énormément le champ de la création variétale. 44 formules hybrides sont déjà obtenues au Congo.

Les descendances hybrides peuvent être très homogènes ou au contraire très hétérogènes. L'hétérosis de la population peut être très élevé ou au contraire concentré sur quelques individus seulement. Dans ce cas, seule la voie végétative sera efficace pour un usage forestier.

L'amélioration des populations hybrides interspécifiques s'achemine vers une voie récurrente réciproque.

\section{La multiplication végétative}

Très bien étudiée et réussie au Congo, elle permet de propager, à grande échelle, les meilleurs individus hybrides.

Les obstacles du vieillissement sont importants, mais l'usage des rejets de souche permet d'utiliser un grand nombre d'individus sélectionnés à l'état adulte. D'autres ne sont pas utilisables. soit parce qu'ils ne rejettent pas, s'enracinent mal ou poussent très mal. Le passage à l'étape industrielle a exigé de nombreuses mises au point. En particulier le cyclage des boutures par rapport aux saisons normales de plantation a été un point très difficile à résoudre au Congo, alors que cela s'est fait sans problèmes à Aracruz, au Brésil, où l'ensemble de toutes les opérations peuvent se dérouler toute l'année en continu, ce qui est exceptionnellement favorable.

De nombreux aspects sont traités tant au niveau expérimental qu'opérationnel. Le problème des tests clonaux et des critères de « juvénilité » est souligné.

\section{La culture clonale}

L'usage des clones d'Eucalyptus tropicaux a été développé à grande échelle depuis 7 à 8 ans au Congo (22000 ha) et au Brésil à Aracruz (90000 ha).

Pour la première fois, en dehors des peupliers, du Cryptomeria et du Paulownia, la ligniculture intensive se développe à l'échelle industrielle, et cela a des répercussions importantes au point de vue économique.

Ces deux exemples permettent d'examiner les problèmes posés par la foresterie clonale et d'en mesurer les risques qui sont doubles: dangers phytosanitaires et réduction de fertilité.

Les risques dus aux parasites sont réels en plantations clonales. Un insecte (Helopeltis bergrothi) est apparu en 1984 dans les plantations clonales du Congo. Les attaques ont été sévères sur jeunes plants. Mais la sélection des clones les moins sensibles est une arme très efficace et très rapide. De même, le nombre de variétés (type d'hybride), le nombre de clones et la disposition des clones sur le terrain ont beaucoup d'importance. L'assistance doit être forte sur le plan cultural, en particulier au niveau de la lutte chimique.

Le maintien de la fertilité est un point important. En sol pauvre, l'usage des clones rustiques est envisagé et l'apport d'engrais est indispensable, de même que le recyclage des écorces, des branches et des feuilles après exploitation. Les gains de production sont considérables et attirent les investisseurs vers ce type de plantations. 
De nombreux problèmes sont évoqués concernant le type de culture à envisager, taillis ou futaie, ainsi que sur l'éthique de la foresterie clonale. Les limites et les arguments essentiels de défense de ce type de culture sont énoncés.

\section{Summary}

\section{Genetic improvement of tropical Eucalyptus : a major contribution to clonal forestry}

The thesis sets out a series of 10 publications from 1971 to 1987 connected with tropical Eucalypt improvement as the main topic. Most of the studies were carried out at Pointe-noire (Congo) at the Centre Technique Forestier Tropical (C.T.F.T.) as well as at the Unité d'Afforestation Industriclle du Congo (U.A.I.C.). A lot of results were also taken from the plantations of Aracruz Florestal S.A. in Brazil (Espirito-Santo).

The documents as a whole were presented as a synthesis including the bibliography available at present. This study consists of seven chapters giving a complete survey of the introduction of the genus in tropical environment and the determination of the conditions for the maximal yield from the best-adapted species, the provenances with the highest output, the most vigorous hybrids and the most advantageous clones.

Various technological aspects were reviewed : exploration, selection, hybridization, vegetative propagation, clonal selection. A real-scale approach of the clonal culture was carefully investigated at Pointe-Noire (25000 ha) as well as at Aracruz (90000 ha) and dangers were estimated. A violent attack by insects was recorded. Thereby, chemical and genetical struggle means were evaluated. Fundamental arguments supporting clonal forestry are developed. 